Дедов Сергей Владимирович, Харченко Екатерина Владимировна

\title{
МОДЕЛИРОВАНИЕ УПРАВЛЕНИЯ ИННОВАЦИОННОЙ ДЕЯТЕЛЬНОСТЬЮ СОЦИАЛЬНО-ЭКОНОМИЧЕСКИХ СИСТЕМ С УЧЕТОМ ВНЕШНИХ ФАКТОРОВ
}

Представленные в статье модели и алгоритмы управления инновачионной деятельностью сочиально-экономических систем построены в соответствии с методологией ВННТ-подхода, позволяющего формализовать в рамках единой системы управления разобщенные представления о внешних компонентах управления инновационной деятельностью: Время, Ннновации, Наука, Технологии. Представлен математический инструиентарий имитационного моделирования систем ситуационного управления инновационной деятельностью с учетом фактора динамики компонент, позволяюиий осуцествлять упрежсающее управление с иелью нейтрализации негативного влияния внешних вызовов и угроз.

Ключевые слова: инновационная деятельность, ресурсное обеспечение, упреждаюиее управление.

\section{Sergey Dedov, Ekaterina Kharchenko MODELLING OF INNOVATION MANAGEMENT OF SOCIO-ECONOMIC SYSTEMS TAKING INTO ACCOUNT EXTERNAL FACTORS \\ Models and algorithms of innovation management of socio-ecological systems presented in the} article are built in accordance with the methodology of the author's approach. This allowed to combine into a single management system separate external components of innovation management: time, innovation, science, technology. Mathematical tools of simulation of systems of situational management of innovation activity are presented. It is built taking into account changing factors. This enables proactive innovation management for the negative impact of external challenges and threats.

Key words: innovation, resources, proactive management.

Bведение/ Introduction. Поиск новых подходов и методов инновационной деятельности и эффективного управления ею представляет широкий научный интерес в связи с необходимостью перманентного использования богатого инновационного потенциала, научных достижений и технологий. Научную интерпретацию теоретико-методологические аспекты и проблемы эффективного управления инновационной деятельностью получили в трудах ряда отечественных и зарубежных авторов. Работы, посвящённые фундаментальным вопросам практики инновационной макроэкономической политики, разработанной на основе институционального проектирования ее структурных элементов, способствовали выявлению закономерностей инновационной деятельности, разработке моделей инновационного развития экономики, институализации условий творческой жизнедеятельности, поиску направлений повышения устойчивости и конкурентоспособности российского бизнеса в ответ на меняющиеся экономические условия [1, 2, 3, 4 и др.].

Теоретико-методологический базис исследования инновационной деятельности, заложенный Б. Мильнером, Й. Шумпетером, Ф. Тейлором, А. Файолем, Г. Эмерсоном и др., развит с учетом институциональных, территориальных и экономических особенностей инновационной деятельности в работах ведущих российских ученых-экономистов $[5,6,7$ и др.]. 
Mameриалы и мemodb// Materials and methods. Предложенный в настоящей статье методологический подход отличается возможностью дифференцированно воздействовать на элементы ресурсного обеспечения управления инновационной деятельностью, что обусловлено объективной необходимостью эффективного управления инновационной деятельностью в теоретико-методологических исследованиях, имеющих практическую направленность. Установление взаимосвязей и зависимостей между внешними компонентами инновационной деятельности, а также координация потенциала механизма ресурсного обеспечения управления инновационной деятельностью проводились с учетом двух детерминирующих моментов: отсутствие директивности в воздействии на указанные компоненты и признание целесообразности их взаимодействия.

Разобщенные представления о внешних компонентах управления инновационной деятельностью (Время, Инновации, Наука и Технологии) формализованы в единую систему в рамках разработанного методологического подхода ВИНТ-подход. Все компоненты по отдельности являются идеальными экзосистемными факторами, обеспечивающими максимальную эффективность при осуществлении управления инновационной деятельностью социально-экономических систем разных уровней. Объединение данных компонент вызвано необходимостью осуществления ресурсного обеспечения управления инновационной деятельностью и локализации возникающих угроз и существующих рисков.

Внешние компоненты в рамках ВИНТ-подхода выступают в роли регуляторов осуществления ресурсного обеспечения управления инновационной деятельностью. Фактор “сжатия пространства-времени», описанный английским экономистом J. Howells [8, с. 226], в условиях глобализации характеризует способность координировать различные функции (исследования, разработки, маркетинг) в едином интегрированном режиме и глобальном масштабе. В настоящее время развитие мирового общества из локально-регионального (случайного) превращается в глобальное (универсальное) условие развития. Диахронное развитие экономического общества становится синхронным. Эпохи, исторически следовавшие друг за другом, в современном мире существуют в едином глобальном пространстве. Небывалый прогресс науки и основанных на ней технологий, неограниченный прогресс человеческих возможностей при формировании инноваций обусловили присутствие феномена сжатия «пружины времени», т. е. одновременного присутствия в настоящем всех предшествующих времён и стадий развития.

Включение компоненты «наука» обусловлено превращением науки в непосредственную производительную силу, что связано с вовлечением в инновационную деятельность знания и информации [9, с. 43]. В процессе эволюции одна система технологических отношений диалектически переходит в другую $[10$, с. 5$]$. В результате определенный набор технологий и инноваций, отвечающий уровню развития социально-экономических систем, составляет динамическую систему технологических отношений.

На рисунке 1 представлена функциональная модель многоуровневой системы управления инновационной деятельностью, где основными объектами, участвующими в управлении инновационной деятельностью, являются организации, отвечающие за мониторинг результатов реализации нормативно-правовых документов, разработку программ инновационной деятельности, контролирующие выполнение целевых установок стратегии инновационного развития страны при одновременном обеспечении безопасности.

В качестве средства анализа систем управления инновационной деятельностью целесообразно использование интеллектуальных систем в их классическом понимании. В алгоритм анализа инновационной деятельности с использованием интеллектуальной системы (рисунок 2) включены базы знаний, которые являются основными элементами социально-экономических систем и предназначены для накопления, хранения и организации доступа к конкретной предметной области, относящейся к факторам групп вызовов. 


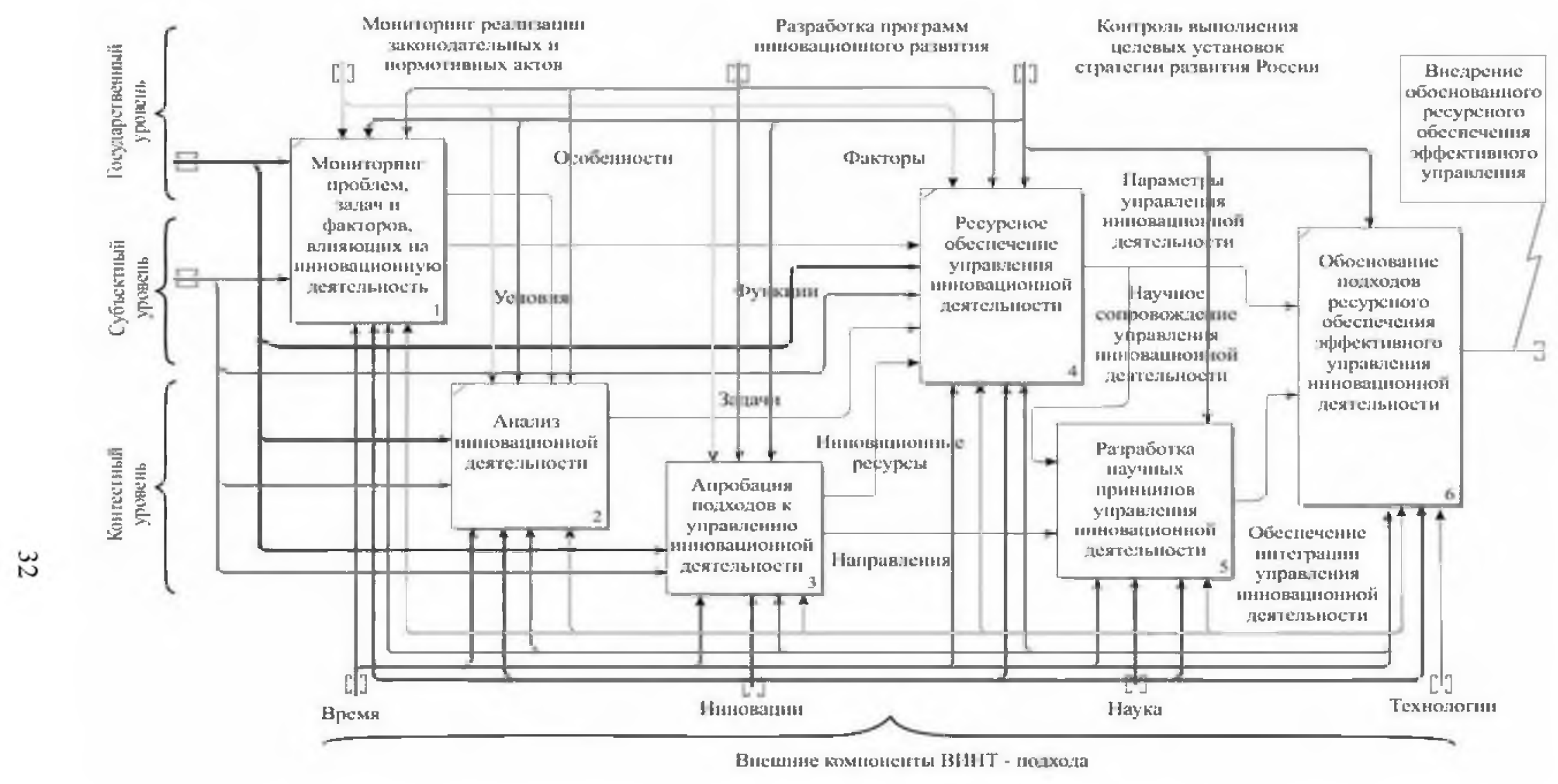

Рис. 1. Функциональная модель многоуровневой системы управления инновационной деятельностью

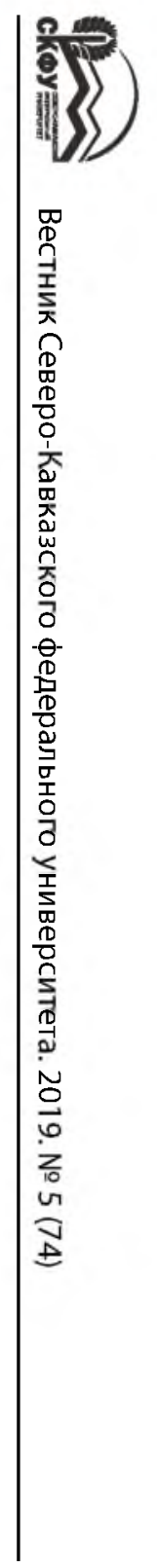


Pезультаты и обсуждение/ Results and discussion. Управление инновационной деятельностью социально-экономической системы является не только важнейшей стратегической, но и достаточно сложной экспертной задачей. В связи с этим управление инновационной деятельностью социально-экономических систем рассматривается нами с позиции взаимодействия внешних компонентов с потенциалами систем (человеческими, научными, технико-технологическими, индустриально-производственными, финансово-экономическими, инвестиционными, информационно-коммуникативными). Приведенные суждения получили отражение в алгоритме управления инновационной деятельностью на основе ВИНТ-подхода (рисунок 2). Для решения задач управления на разных уровнях необходимо обеспечить возможность:

- $\quad$ учета свойств, признаков и связей с точки зрения будущих задач всех участвующих в инновационной деятельности объектов;

- использования фильтров, отсеивающих неинформативные события;

- анализа объектов, вовлекаемых в инновационную деятельность с любого временного интервала;

- идентификации условий, при которых субъекты и объекты инновационной деятельности характеризуются необъективно;

- детализации базовых характеристик предметной области - понятийной структуры, отношений между принятыми моделями и способов рассуждений.

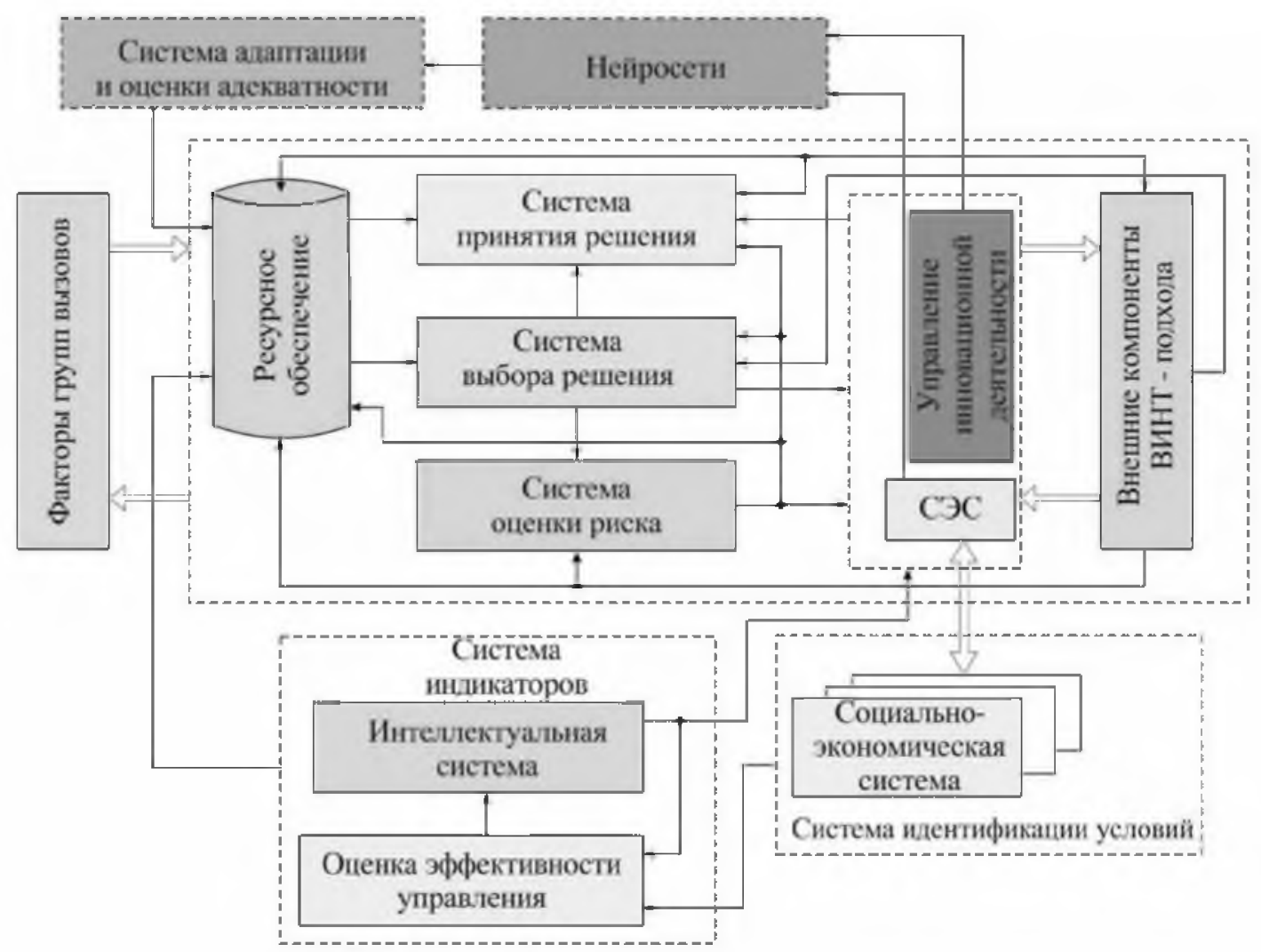

Рис. 2. Алгоритм анализа инновационной деятельности с использованием интеллектуальной системы

Обозначим понятия, относящиеся к группе управления инновационной деятельностью, как $A=\left\{A_{1}, A_{2}\right\}$, где $A_{1}$ - простые, а $A_{2}$ - сложные понятия. Отношения объектов при осуществлении инновационной деятельности обозначим как $B=\left\{B_{1}, B_{2}\right\}$, где $B_{1}$ - незначительное число отношений, 
связывающих понятия данной предметной области управления и $B_{2}-$ значительное число отношений. Виды логического вывода обозначим как $C=\left\{C_{1}, C_{2}, C_{3}\right\}$, где $C_{1}-$ индуктивный вывод; $C_{2}$ - дедуктивный вывод; $C_{3}-$ абдуктивный вывод.

Формально правила выбора моделей представления компонентов запишем следующим образом:

$$
\left\{\begin{array}{c}
A_{1} v B_{1} v C_{1} \rightarrow M_{5} ; \\
A_{1} v B_{1} v C_{2} \rightarrow M_{1} ; \\
A_{1} v B_{1} v C_{3} \rightarrow M_{1} M_{2} ; \\
A_{2} v B_{1} v C_{1} \rightarrow M_{2}, M_{23} ; \\
A_{2} v B_{1} v C_{2} \rightarrow M_{6} ; \\
A_{2} v B_{1} v C_{3} \rightarrow M_{2} ; \\
A_{2} v B_{2} v C_{1} \rightarrow M_{23} ; \\
A_{2} v B_{2} v C_{2} \rightarrow M_{3} ; \\
A_{2} v B_{2} v C_{3} \rightarrow M_{2} ; \\
A_{1} v B_{2} v C_{1} \rightarrow M_{4} ; \\
A_{1} v B_{2} v C_{2} \rightarrow M_{4}, M_{43} ; \\
A_{1} v B_{2} v C_{3} \otimes M_{3}, M_{2},
\end{array}\right.
$$

В свою очередь, модели обозначим $M=\left\{M_{1}, M_{2}, M_{3}, M_{4}, M_{5}, M_{6}\right\}$, где $M_{1}$ - логическая модель; $M_{2}$ - сетевая модель; $M_{3}$ - фреймовая модель; $M_{4}$ - продукционная модель; $M_{5}-$ индуктивная модель; $M_{6}$ - тезаурусные модели. Следует отметить, что множество $M$ не отражает всей полноты моделей представления знаний. Поэтому нами введены обозначения смешанных моделей, где $M_{23}$ - сеть фреймов, $M_{43}$ - продукционно-фреймовая модель.

Тогда правила можно сформулировать следующим образом:

$$
\left\{\begin{array}{l}
D_{1} v E_{1} \rightarrow M_{1} ; \\
D_{1} v E_{2} \rightarrow M_{4} \\
D_{2} v E_{1} \rightarrow M_{2} \\
D_{2} v E_{2} \rightarrow M_{3}
\end{array}\right.
$$

Кроме того, при окончательном выборе модели представления компонентов предлагаем учесть их «глубину» $D$ и «доступность» $E$. По «глубине» компоненты разделим на поверхностные $D_{1}$ и глубинные $D_{2}$, а по «доступности» - на общие $E_{1}$ и уникальные $E_{2}$.

Применительно к предметной области управления инновационной деятельностью проводимых исследований необходимо определить базовые характеристики для всех компонентов в рамках ВИНТ-подхода. Таким образом, согласно (1), для формализации управления инновационной деятельностью необходимо представлять компоненту преимущественно в виде сетей фреймов, являющихся наиболее универсальным для решения поставленной задачи эффективного управления инновационной деятельностью.

Управление инновационной деятельностью социально-экономических систем с определенной степенью условности можно разделить на три ситуационных вида (уровня): управление при изменении отдельной компоненты; управление по изменению двух компонент, управление по изменению группы совокупности компонентов. Управление инновационной деятельностью в формализованном виде для удобства представим в виде кортежа (3):

$$
\Theta=\langle K, S, T>\text {, }
$$

где $K$ - рассматриваемая внешняя компонента; $S$ - ситуационный вид управления социально-экономической системой; $T$ - период времени действия хотя бы одной компоненты. 
Модель ситуационного управления инновационной деятельностью (3) представим в виде графа

$$
S=\left(W^{s}, R^{s w}, T^{s w}, P^{s w}\right),
$$

где $\mathrm{W}_{\mathrm{n}}^{\mathrm{s}}$ - уровень управления инновационной деятельностью социально-экономических систем,

$$
\mathrm{W}_{\mathrm{n}}^{\mathrm{s}}=\left\{\mathrm{W}_{1}^{1}, \ldots \mathrm{W}_{\mathrm{i}}^{\mathrm{s}-1}, \ldots \mathrm{W}_{\mathrm{n}}^{\mathrm{s}}\right\},
$$

где $i=\overline{1, n}$ - номер объекта, участвующего в инновационной деятельности; $\mathrm{R}_{j}^{\mathrm{sw}}-$ ранг этапа управления,

$$
\mathbf{R}^{\mathrm{sw}}=\left\{\mathbf{R}_{2}^{\mathrm{s1}}, \mathbf{R}_{3}^{\mathrm{s} 2}, \ldots, \mathbf{R}_{\mathrm{j}}^{\mathrm{s}(\mathrm{n}-1)}\right\},
$$

где $j=\overline{2, n}, i<j ; \quad \mathrm{T}^{\text {sw }}$ - время, необходимое для принятия решения по каждой внешней компоненте управления инновационной деятельностью социально-экономических систем

$$
\mathrm{T}^{\mathrm{sw}}=\left\{\mathrm{T}^{\mathrm{s1}}, \mathrm{T}^{\mathrm{s} 2}, \mathrm{~T}^{\mathrm{si}} \ldots, \mathrm{T}^{\mathrm{sn}}\right\},
$$

$P^{s w}$ - обобщенное значение весовых коэффициентов,

$$
P^{s w}=\left\{P_{i}^{s w}, P_{2}^{s w}, P_{i}^{s w} \ldots, P_{n}^{s w}\right\},
$$

где $P_{n}^{s w}$ - весовые коэффициенты, оценивающие выбранный уровень управления инновационной деятельностью социально-экономических систем, находящиеся в пределах $0 \leq P_{m}^{s w} \leq 1$.

Данные соотношения позволяют изобразить управление инновационной деятельностью с помощью графа, где узлы - объекты соответствующего вида управления, участвующие в инновационной деятельности (4), дуги отражают ранг этапа управления социально-экономических систем (6), показывающий их приоритет на рассматриваемом уровне. Скаляры, приписанные вершинам для каждой ситуации, показывают приоритет данного объекта перед другими для каждой ситуации и время (7), которое необходимо для принятия решения по каждому внешнему компоненту управления инновационной деятельностью социально-экономической системы (рисунок 3 ).

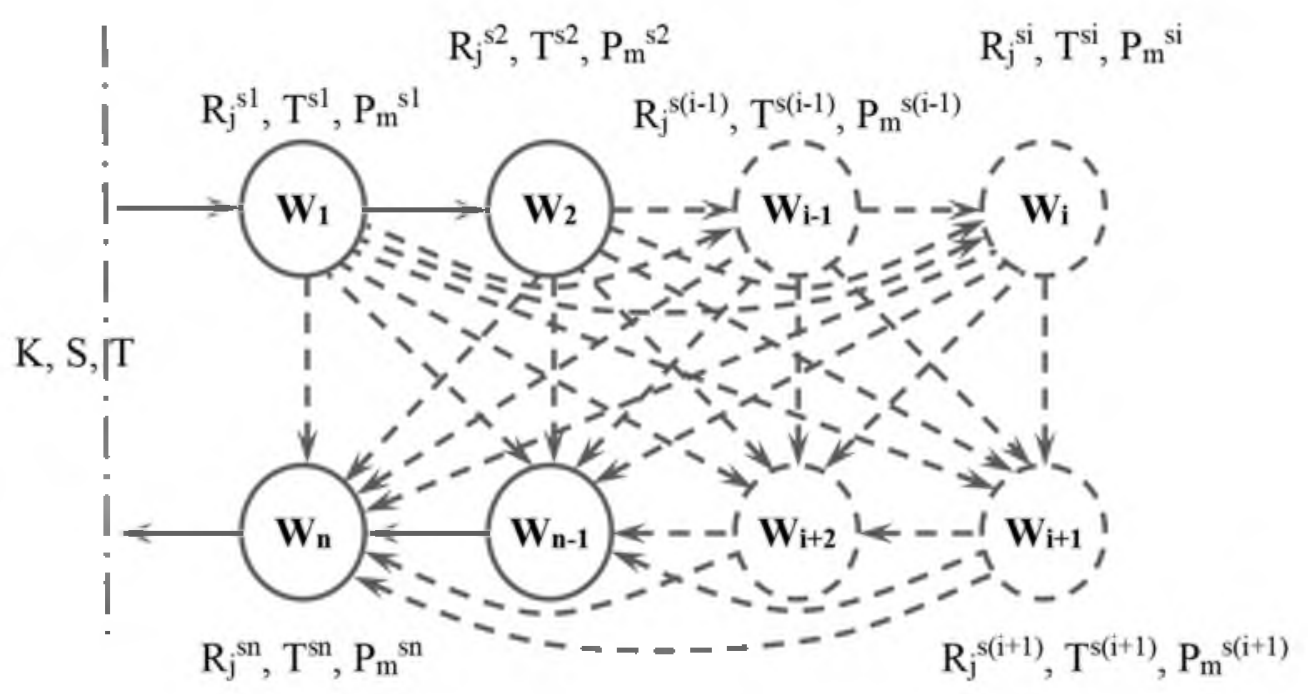

Рис. 3. Имитационная модель системы управления инновационной деятельностью социально-экономических систем с учетом внешних факторов

$$
W^{s}=\left(\mathrm{Q}^{\mathrm{sw}}, \mathrm{R}^{\mathrm{swq}}, \mathrm{T}^{\mathrm{swq}}, \mathrm{P}^{\mathrm{swq}}\right),
$$

где $\mathrm{Q}^{\mathrm{sw}}$ - объекты социально-экономических систем, 


$$
\mathrm{Q}^{\mathrm{sw}}=\left\{\mathrm{Q}_{1}^{\mathrm{sw}}, \mathrm{Q}_{1}^{\mathrm{sw}}, \ldots, \mathrm{Q}_{\mathrm{b}}^{\mathrm{sw}}\right\},
$$

где $i=\overline{1, b}$ - номер объекта оцениваемого уровня; $\mathrm{R}^{\text {swq }}$ - ранг этапа управления объектами социально-экономических систем,

$$
\mathbf{R}^{\mathrm{swq}}=\left\{\mathbf{R}_{2}^{\mathrm{sw1}}, \mathbf{R}_{3}^{\mathrm{sw1}}, \ldots, \mathbf{R}_{\mathrm{j}}^{\mathrm{sw}(\mathrm{b}-1)}\right\},
$$

где $i=\overline{1, b}$ - номера объектов социально-экономических систем, $j=\overline{2, b}, i<j$; $\mathrm{T}^{\text {swq }}$ - время, необходимое для принятия решения по каждой внешней компоненте управления объектами социально-экономических систем, участвующими в инновационной деятельности

$$
\mathrm{T}^{\mathrm{swq}}=\left\{\mathrm{T}^{\mathrm{swl}}, \mathrm{T}^{\mathrm{swi}}, \ldots, \mathrm{T}^{\mathrm{swb}}\right\},
$$

где $i=\overline{1, b}$ - номер объектов оцениваемого уровня социально-экономических систем; $P_{t q}^{s w q}-$ весовые коэффициенты, оценивающие выбранный уровень управления инновационной деятельностью объектов социально-экономических систем

$$
\begin{gathered}
P^{s}=\left\{P_{+}^{s w q}, P_{i}^{s w q}, P_{m}^{s w q}\right\}, \\
P_{m}^{s w q}=0 \mid E_{m}^{s w}=1, \\
0<P_{m}^{s w q} \leq 1 \mid E_{m}^{s w}=0,
\end{gathered}
$$

где $i=\overline{1, m}$ - номер объекта социально-экономических систем, $E_{m}^{s w}-$ результат управляющего воздействия.

Практическое использование представленного математического аппарата моделирования влияния внешних факторов на управление инновационной деятельностью социально-экономических систем в рамках ВИНТ-подхода позволило сделать ряд выводов: 1) использование ВИНТ-подхода позволяет заблаговременно выявлять необходимость проведения упреждающих мероприятий; 2) возможность постоянного мониторинга экономических, технологических, социологических и экологических трендов; 3) эффективное управление инновационной деятельностью с учетом внешних факторов зависит от ее ресурсного обеспечения. Однако, актуализация содержательной части ВИНТ-подхода, прикладной характер обоснования роли внешних факторов в управлении инновационной деятельностью социально-экономических систем еще не гарантируют стабильность результатов. Реализация данного подхода требует проведения углубленного методологического анализа ресурсного обеспечения управления инновационной деятельностью.

Заключение/Conclusion. Предложенная функциональная модель многоуровневой системы управления инновационной деятельностью отличается структурированием компонентов ресурсного обеспечения и позволяет учитывать дихотомичность исторических, территориальных и временных особенностей, позволяет своевременно нейтрализовать вызовы и угрозы. Своевременная идентификация состояний социально-экономических систем невозможна без контроля, диагностирования и прогнозирования экономических последствий, оперативного выполнения анализа большого объема информации при проведении оценки факторов групп вызовов и качественно-количественной оценки реальных экономических последствий. Упреждающее управление инновационной деятельностью на основе ВИНТ-подхода осуществляется на основе выбора вариантов локализации последствий ситуаций (угрозы, санкции, кризисы) с позиции институционального подхода как основного условия, обеспечивающего совершенствование форм и способов исследования управления инновационной деятельностью в экономических системах.

\section{ЛИТЕРАТУРА И ИНТЕРНЕТ-РЕСУРСЫ}

1. Стратегический ответ России на вызовы нового века / Под общ. ред. Л. И. Абалкина. Москва : Издво «Экзамен». 2004. -608 с. 
2. Рисин И. Е., Трешевский Ю. И., Эйтингон В. Н. Ключевые задачи научно-технологической политики и подходы к их решению // Вестник Воронежского государственного университета. Серия: Экономика и управление. 2014. - № 2. - С. 134-138.

3. Колмыкова Т. С., Широкова Л. В., Галахов Д. И. Исследование вклада наукоемких и высокотехнологичных производств в обеспечение экономического роста: эмпирический аспект // Национальные интересы: приоритеты и безопасность. 2014. - Т. 10. - № 9 (246). - С. 2-8.

4. Управление развитием высокотехнологичных секторов в формировании воспроизводственных контуров инновационной экономики / Емельянов С. Г., Харченко Е. В., Широкова Л. В., Алпеева Е. А., Ситникова Э. В., Галахов Д. И., Колмыкова Т. С. -Курск, 2013. - 324 с.

5. Дятлов С. А. Информационная модель трансформации экономических систем. Санкт-Петербург : СПб. ун-т экономики и финансов. - 1996. - 18 с.

6. Иноземцев В. Л. Современное постиндустриальное общество: природа, противоречия, перспективы: Учеб. пособие для студентов вузов. Москва: Логос. $-2000 .-304$ с.

7. Татаркин А. И. Модернизационное обновление российского пространства на основе инновационных инициатив // Регион : Экономика и социология, 2016. - № 1. - С. 6-33.

8. Howells J. R. Emerging global strategies in innovation management // The impact of globalization on Europe`s firm and industries. - L.; NY. : Printer pub1., 1993. - pp. 219-228.

9. Кузык Б. Н., Агеев А. И., Доброчеев А. В. и др. Россия в пространстве и времени: История будушего. Москва : Ин-т эконом.стратегий. -2004 . -335 с.

10. Федулова Л. И. Экономическая природа технологий и технологического развития // Экономическая история, 2006. - № 3. - С. 3-19.

\section{REFERENCES AND INTERNET RESOURCES}

1. Strategicheskij otvet Rossii na vyzovy novogo veka (Russia's Strategic Response to the Challenges of the New Century), Pod obshh. red. L. I. Abalkina, M.: Izd-vo «Jekzamen», 2004, 608 s.

2. Risin I. E., Treshhevskij Ju.I., Jejtingon V.N. Kljuchevye zadachi nauchno-tehnologicheskoj politiki i podhody $\mathrm{k}$ ih resheniju (Key science and technology policy objectives and approaches), // Vestnik Voronezhskogo gosudarstvennogo universiteta. Serija: Jekonomika i upravlenie, 2014. - №. 2, S. 134-138.

3. Kolmykova T. S., Shirokova L. V., Galahov D. I. Issledovanie vklada naukoemkih i vysokotehnologichnyh proizvodstv v obespechenie jekonomicheskogo rosta: jempiricheskij aspect (Research on the contribution of knowledge-intensive and high-tech industries to economic growth: an empirical dimension), // Nacional'nye interesy: prioritety i bezopasnost', 2014. - T. 10, - № 9 (246). S. 2-8.

4. Upravlenie razvitiem vysokotehnologichnyh sektorov $\mathrm{v}$ formirovanii vosproizvodstvennyh konturov innovacionnoj jekonomiki (Management of development of high-tech sectors in formation of reproductive circuits of innovation economy), Emel'janov S. G., Harchenko E. V., Shirokova L. V., Alpeeva E. A., Sitnikova Je. V., Galahov D. I., Kolmykova T. S. - Kursk, 2013, - 324 s.

5. Djatlov S. A. Informacionnaja model' transformacii jekonomicheskih system (Information model of transformation of economic systems), $\mathrm{S}$. $-\mathrm{Pb}$.: $\mathrm{SPb}$. un-t jekonomiki i finansov. $-1996,-18 \mathrm{~s}$.

6. Inozemcev V. L. Sovremennoe postindustrial'noe obshhestvo: priroda, protivorechija, perspektivy (Modern post-industrial society: nature, contradictions, perspectives): Ucheb. posobie dlja studentov vuzov, Moskva : Logos, 2000. - 304 p.

7. Tatarkin A. I. Modernizacionnoe obnovlenie rossijskogo prostranstva na osnove innovacionnyh iniciativ (Modernization of Russian space on the basis of innovative initiatives), // Region: Jekonomika i sociologija, 2016. - № 1. - S. 6-33.

8. Howells J. R. Emerging global strategies in innovation management, The impact of globalization on Europe's firm and industries. - L.; NY. : Printer pub1., 1993. - S. 219-228.

9. Kuzyk B. N., Ageev A. I., Dobrocheev A. V. i dr. Rossija v prostranstve i vremeni: Istorija budushhego (Russia in Space and Time: History of the Future), M.: In-t jekonom.strategij, 2004. - $335 \mathrm{~s}$.

10. Fedulova L. I. Jekonomicheskaja priroda tehnologij i tehnologicheskogo razvitija (Economic nature of technology and technological development), Jekonomicheskaja istorija, 2006. - №. 3. - S. 3-19. 


\section{СВЕДЕНИЯ ОБ АВТОРАХ}

Дедов Сергей Владимирович, кандидат физико-математических наук, доцент, Военный учебно-научный центр Военно-воздушных сил «Военно-воздушная академия им. проф. Н. Е. Жуковского и Ю. А. Гагарина», г. Воронеж. E-mail: generdeser@ $a$ bk.ru

Хариенко Екатерина Владимировна, доктор экономических наук, профессор, профессор кафедры экономики, управления и аудита, Юго-Западный государственный университет, г. Курск. E-mail: swsu_ buaia@mail.ru

\section{INFORMATION ABOUT AUTHORS}

Sergey $V$. Dedov, candidate of physical and mathematical Sciences, associate Professor, Military training and research center of the Air force «Air force Academy prof. N. E. Zhukovsky and Y. A. Gagarin», Voronezh. E-mail: generdeser $a$ bk.ru

Ekaterina V. Kharchenko, doctor of Economics, Professor, Professor of the Department of Economics, management and audit, Southwest state University, Kursk. E-mail: swsu_buaia@mail.ru 